\title{
Multifunctional molybdenum disulfide flash memory using a PEDOT:PSS floating gate
}

Seongin Hong ${ }^{1}$, Junwoo Park', Jung Joon Lee $\mathbb{D}^{1}$, Sunjong Lee ${ }^{2}$, Kyungho Yun², Hocheon Yoo $\mathbb{1}^{3}$ and Sunkook Kim (1)

\begin{abstract}
Two-dimensional transition metal dichalcogenide materials (TMDs), such as molybdenum disulfide $\left(\mathrm{MoS}_{2}\right)$, have been considered promising candidates for future electronic applications owing to their electrical, mechanical, and optical properties. Here, we present a new concept for multifunctional $\mathrm{MoS}_{2}$ flash memory by combining a $\mathrm{MoS}_{2}$ channel with a PEDOT:PSS floating layer. The proposed $\mathrm{MOS}_{2}$ memory devices exhibit a switching ratio as high as $2.3 \times 10^{7}$, a large memory window $(54.6 \pm 7.80 \mathrm{~V})$, and high endurance (>1,000 cycles). As the PEDOT:PSS film enables a lowtemperature solution-coating process and mechanical flexibility, the proposed P-memory can be embedded on a polyimide substrate over a rigid silicon substrate, offering high mechanical endurance (over 1,000 cycle bending test). Furthermore, both $\mathrm{MOS}_{2}$ and PEDOT:PSS have a bandgap that is desirable in optoelectronic memory operation, where charge carriers are stored differently in the floating gate depending on light illumination. As a new application that combines photodiodes and memory functions, we demonstrate multilevel memory programming based on light intensity and color.
\end{abstract}

\section{Introduction}

With the advent of the fourth industrial revolution, consumer electronics are in dire need of versatility beyond conventional electronic functions ${ }^{1-4}$. For example, conventional memory technology requires high-performance memory characteristics, such as fast switching, long-term retention, and high endurance ${ }^{5-8}$. On the other hand, current electronics demand the development of multifunctional memory devices with characteristics such as mechanical flexibility ${ }^{9-11}$, multilevel storage ${ }^{12,13}$, neuromorphic functions ${ }^{14,15}$, and tunable memory operation ${ }^{16}$, along with the conventional expectation of excellent memory features. However, existing silicon-based memory processes and structures have limitations in developing new features when the multifunctional memory

\footnotetext{
Correspondence: Hocheon Yoo (hyoo@gachon.ac.kr) or

Sunkook Kim (seonkuk@skku.edu)

${ }^{1}$ School of Advanced Materials Science and Engineering Sungkyunkwan University, Sunwon 440-746, Korea

${ }^{2}$ Korea Institute of Industrial Technology, Cheonan 31056, Republic of Korea Full list of author information is available at the end of the article

These authors contributed equally: Seongin Hong, Junwoo Park
}

deviates from the conventional complementary metal-oxide-semiconductor process frame. Therefore, with the development of new materials, the development of new electronic device concepts is crucial.

Meanwhile, as emerging semiconductor materials, transition metal dichalcogenides (TMDs) have attracted considerable attention ${ }^{17-21}$. TMDs have the potential to provide multifunctional material platforms due to a variety of physical properties, including their atomically thin nature ${ }^{22}$, van der Waals interface ${ }^{23}$, and controllable energy band structure ${ }^{24-26}$. Moreover, TMDs have shown the possibility of new electronic device features such as gate-tunable memristic behavior ${ }^{16,27}$, neuromorphic functions ${ }^{28}$, and optoelectronic properties ${ }^{29,30}$.

Here, we present multifunctional nonvolatile memory using a molybdenum disulfide $\left(\mathrm{MoS}_{2}\right)$ semiconductor and a poly(3,4-ethylenedioxythinophene):poly(styrene sulfonate) (PEDOT:PSS) ${ }^{31}$ floating gate (P-memory). The combination of the $\mathrm{MoS}_{2}$ semiconductor and PEDOT:PSS floating gate provides a switching current ratio as high as $2.3 \times 10^{7}$, a large memory window up to $62 \mathrm{~V}$, excellent

\section{(c) The Author(s) 2021}

(c) (i) Open Access This article is licensed under a Creative Commons Attribution 4.0 International License, which permits use, sharing, adaptation, distribution and reproduction cc) in any medium or format, as long as you give appropriate credit to the original author(s) and the source, provide a link to the Creative Commons license, and indicate if changes were made. The images or other third party material in this article are included in the article's Creative Commons license, unless indicated otherwise in a credit line to the material. If material is not included in the article's Creative Commons license and your intended use is not permitted by statutory regulation or exceeds the permitted use, you will need to obtain permission directly from the copyright holder. To view a copy of this license, visit http://creativecommons.org/licenses/by/4.0/. 


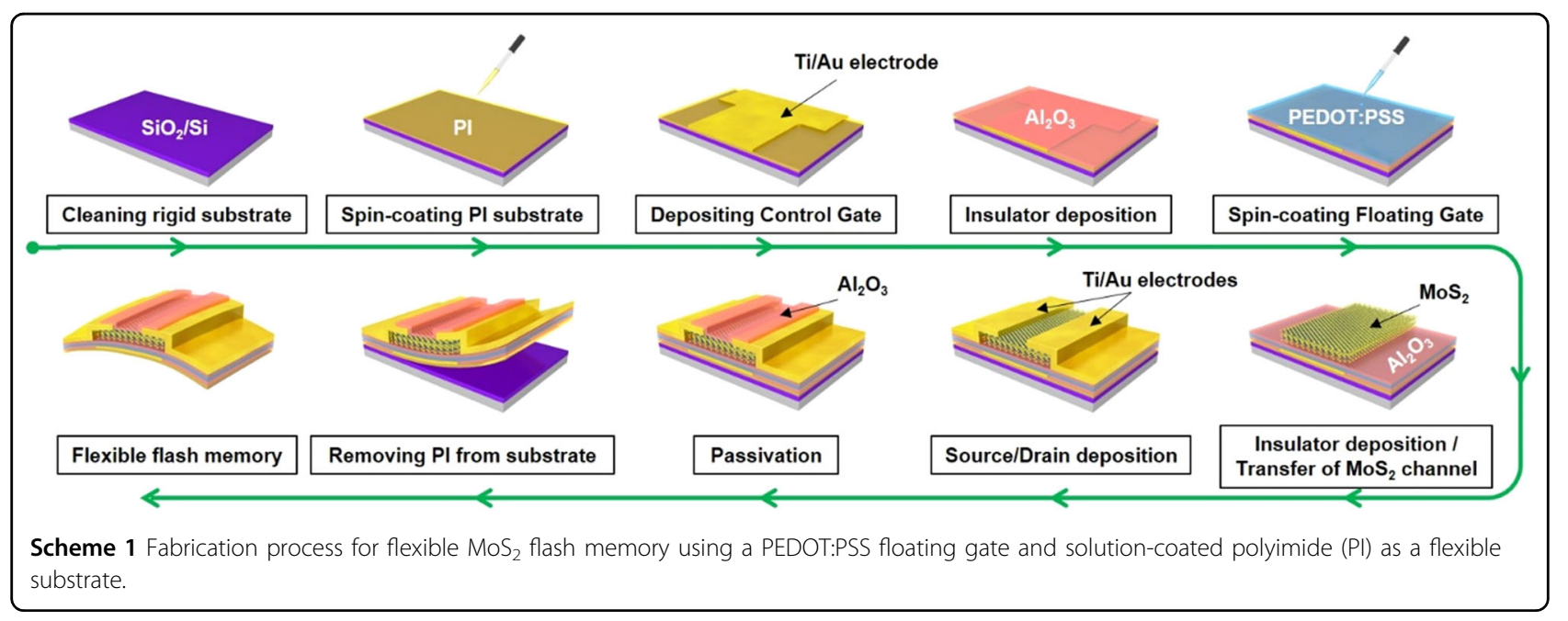

endurance (>1000 cycles), and a long retention time ( $>2000 \mathrm{~s}$ ). We also investigate device-to-device variation by examining $30 \mathrm{P}$-memory devices fabricated with the same fabrication process. This process results in uniform memory operation properties with average $I_{\text {erase }} / I_{\text {program }}$ of $2.18 \times 10^{6} \pm 4.77 \times 10^{6}$, memory window of $54.3 \mathrm{~V} \pm$ $7.80 \mathrm{~V}$, threshold voltage under the programmed state $V_{T H}$, programmed of $16.40 \pm 8.25 \mathrm{~V}$, and threshold voltage under the erased state $V_{T H}$, erased of $-40.53 \pm 5.87 \mathrm{~V}$. Furthermore, the proposed P-memory has photoprogrammable memory behavior, as photoinduced charge carriers contribute to programming and erasing operations in P-memory. As a result, we demonstrate photosensing with nonvolatile memory behavior to distinguish illumination light intensity and color. This information is stored directly inside the floating gate. Finally, we implement P-memory embedded onto a flexible polyimide (PI) substrate, as both $\mathrm{MoS}_{2}$ and PEDOT:PSS can be simply deposited regardless of the substrate type. The fabricated P-memory on the PI substrate exhibits excellent memory operation $\left(I_{\text {erase }} / I_{\text {program }}=1.07 \times 10^{6}\right)$ with a high mechanical endurance (over 1000 cycle bending test). This result suggests that the multifunctional characteristics of a flash memory device can be obtained from a new material combination of layered $\mathrm{MoS}_{2}$ and PEDOT: PSS polymer in a floating-gate transistor structure.

\section{Materials and methods Device fabrication}

The device fabrication process is schematically shown in Fig. S1. A Si/ $/ \mathrm{SiO}_{2}$ substrate was treated with $\mathrm{O}_{2}$ plasma for $5 \mathrm{~min}$. A synthesized highly resistive PEDOT:PSS solution was spin-coated on the substrate at $8000 \mathrm{rpm}$ for $60 \mathrm{~s}$ through a $0.45 \mu \mathrm{m}$ syringe filter. The thin film was then thermally annealed on a hot plate at $150^{\circ} \mathrm{C}$ for $10 \mathrm{~min}$. After floating-gate formation on the $\mathrm{Si} / \mathrm{SiO}_{2}$ substrate, $\mathrm{Al}_{2} \mathrm{O}_{3}$ of $80 \mathrm{~nm}$ thickness as the tunneling dielectric layer (TDL) was deposited on the PEDOT:PSS floating-gate layer using ALD. To utilize $\mathrm{MoS}_{2}$ flakes as the channel, mechanically exfoliated $\mathrm{MoS}_{2}$ flakes were transferred onto the TDL by peeling them from a bulk crystal using Scotch tape. To complete the P-memory device fabrication, Ti/Au $(20 \mathrm{~nm} / 100 \mathrm{~nm})$ source/drain electrodes, respectively, were patterned by photolithography using AZ GXR-601 photoresist, followed by E-beam evaporation and an etching process. Next, a $20 \mathrm{~nm}$-thick $\mathrm{Al}_{2} \mathrm{O}_{3}$ passivation layer (PL) was deposited on the P-memory device by using ALD. For flexible memories, a flexible substrate was prepared by spin coating solution-based PI on a cleaned $\mathrm{SiO}_{2} / \mathrm{Si}$ substrate (3000 rpm for $30 \mathrm{~s})$. Then, the flexible device was fabricated following the same process as shown in Scheme 1. As the last step, the flexible memory was peeled off by carefully separating the edges of the flexible memory and rigid substate with tweezers, which completed the flexible memory fabrication process.

\section{Synthesis of the highly resistive PEDOT:PSS solution}

Poly(4-styrene sulfonate) (PSS) was used as a dispersant and dopant for PEDOT. The weight ratio of PEDOT:PSS was controlled to be 1:7.5. To synthesize the PEDOT:PSS dispersion, PSS $(18.75 \mathrm{~g})$ was added to distilled water $(1000 \mathrm{~g})$, and the mixture was stirred for $30 \mathrm{~min}$. Then, nitrogen gas (99.999\%) was bubbled through the solution for $60 \mathrm{~min}$ at a rate of $3 \mathrm{~L} \mathrm{~min}^{-1}$ to prevent oxidation by the dissolved oxygen in the water. To this solution, the EDOT monomer $\left(2.50 \mathrm{~g}, 1.76 \times 10^{-2} \mathrm{~mol}\right)$ was added and stirred with a mechanical stirrer for $30 \mathrm{~min}$. The synthesis of the PEDOT:PSS dispersion was carried out using an $\mathrm{Fe}^{3+}$-catalyzed oxidative polymerization process. The oxidizing agents iron(III) sulfate $\left(0.035 \mathrm{~g}, 8.79 \times 10^{-5} \mathrm{~mol}\right)$ and sodium persulfate $\left(5.02 \mathrm{~g}, 2.1 \times 10^{-2} \mathrm{~mol}\right)$ were dissolved in $100 \mathrm{~mL}$ of distilled water by sonication and added to the reaction solution. Polymerization was 
Table 1 Previously reported solvent exchange processes for water-based PEDOT:PSS solutions.

\begin{tabular}{|c|c|c|c|c|}
\hline Method & Solvent & $\begin{array}{l}\text { Stability of re- } \\
\text { dispersion }\end{array}$ & Problems (limitations) & Ref. \\
\hline Mixture \& Evaporation & NMP, DMAC, DMF, etc. & Not verified & $\begin{array}{l}\text { - Limited solvent (High b.p }>150^{\circ} \mathrm{C} \text { ) } \\
\text { coating drying temperature requirement, poor } \\
\text { - Environmentally restricted materials (NMP, DMAC, } \\
\text { DMF) by EU, 'REACH' (2018.10.31 Present) }\end{array}$ & 19 \\
\hline Mixture $(3: 1 \mathrm{v} / \mathrm{v})$ & DMSO, DMF, THF & Not verified & - Simple mixing, not solvent exchange & 20 \\
\hline $\begin{array}{l}\text { Synthesis of Alkyl } \\
\text { derivative PEDOT }\end{array}$ & Chloroform, DMF & Good & $\begin{array}{l}\text { - Far less conductive } \\
\text { - Very complex, small production }\end{array}$ & 21 \\
\hline $\begin{array}{l}\text { Synthesis of PEDOT:PSS in } \\
\text { organic solvent }\end{array}$ & $\begin{array}{l}\text { Methanol, THF, } \\
\text { Toluene, etc. }\end{array}$ & Moderate & $\begin{array}{l}\text { - Far less conductive } \\
\text { - Complex and complicated }\end{array}$ & 22 \\
\hline Ultrafiltration & $\begin{array}{l}\text { All with high } \partial \mathrm{H} \\
(>19.4) \text { such as } \mathrm{EtOH}, \\
\mathrm{EG} \text {, etc. }\end{array}$ & Very Good & $\begin{array}{l}<\text { Advantages }> \\
\text { - Various solvents (both high and low b.p) } \\
\text { - Highly conductive (no loss of conductivity) } \\
\text { - Simple, fast, and conducive to mass production }\end{array}$ & This work \\
\hline
\end{tabular}

performed for $40 \mathrm{~h}$ at $25^{\circ} \mathrm{C}$ with bubbling nitrogen gas. After polymerization of the PEDOT:PSS dispersion, the product was mixed with $500 \mathrm{~mL}$ of a mixture of cation and anion ion exchange resin for $1 \mathrm{~h}$ and filtered with a $30 \mu \mathrm{m}$ mesh filter. Table 1 shows previous reports about solvent exchange processes for water-based PEDOT:PSS solutions.

\section{Device characterization}

The memory operation characteristics of $\mathrm{MoS}_{2}$ flash memory devices were determined with a Keithley 4200SCS semiconductor characterization system equipped with a probe station in the dark under ambient atmosphere. For determining the photoinduced memory operation properties, illumination sources of $638 \mathrm{~nm}$ and $405 \mathrm{~nm}$ (MCLS1, Thorlabs) and $532 \mathrm{~nm}$ (MGL-FN-532, $\mathrm{CNI}$ ) were used. The incident power densities of the illumination light were measured by a laser power meter (PM100A, Thorlabs). To perform a cyclic bending test, the flexible $\mathrm{MoS}_{2}$ flash memory was loaded onto a multimodal bending tester, which repeated the sequence of a tensile stress $(1 \mathrm{~s}) /$ flat $(1 \mathrm{~s})$ cycle 1000 times. The surface morphology and thickness profile of $\mathrm{MoS}_{2}$ were measured using atomic force microscopy (XE7, Park Systems).

\section{Results and discussion}

Flash memory structure based on $\mathrm{MoS}_{2}$ and PEDOT:PSS

First, we fabricated the P-memory on a rigid $\mathrm{SiO}_{2} / \mathrm{Si}$ substrate. The memory device is composed of multilayer $\mathrm{MoS}_{2}$ (thickness of $94.09 \mathrm{~nm}$ ) as the channel; PEDOT:PSS of $80 \mathrm{~nm}$ thickness as the floating gate; $80 \mathrm{~nm}$ - and $40 \mathrm{~nm}$ thick $\mathrm{Al}_{2} \mathrm{O}_{3}$ as a TDL and a $\mathrm{PL}$, respectively; Ti/Au
$(20 \mathrm{~nm} / 100 \mathrm{~nm})$ as source/drain electrodes, respectively; and a $300 \mathrm{~nm} \mathrm{SiO}_{2} / \mathrm{Si}$ substrate as a blocking dielectric layer (BDL) and a control gate (CG) (Fig. 1a). Further details of the fabrication process are provided in Fig. S1, and thickness scans are shown in Fig. S2.

We spin-coated a PEDOT:PSS layer on top of a BDL $\mathrm{SiO}_{2}$ layer, and thereby, the PEDOT:PSS acted as a charge trapping layer for data storage operation (Fig. 1b, c). To understand the effects of the floating-gate layer, we characterized the $\mathrm{MoS}_{2}$ transistor depending on the existence of the PEDOT:PSS floating gate using the same device structure and fabrication conditions. The $\mathrm{MoS}_{2}$ transistor without the PEDOT:PSS floating gate exhibited transfer characteristics without $I-V$ hysteresis (Fig. 1d). On the other hand, the P-memory with the PEDOT:PSS floating gate exhibited transfer characteristic with a large memory window of $54.6 \mathrm{~V} \pm 7.80 \mathrm{~V}$, indicating that the PEDOT:PSS floating gate between the TDL and BDL layers acts as a charge storage layer (Fig. 1e and Fig. S3), showing a switching ratio as high as $2.3 \times 10^{7}$.

\section{Electrical characteristics of the P-memory}

To investigate the endurance of the P-memory, we performed 1000 consecutive sweeps of programming and erasing operations $\left(V_{\text {program }}=60 \mathrm{~V}\right.$ and $V_{\text {erase }}=-60 \mathrm{~V}$ ) (Fig. 1f). The measured endurance of the P-memory exhibited stable programming and erasing operations with average $I_{\text {erase }}$ of $1.4 \pm 0.25 \mu \mathrm{A}, I_{\text {program }}$ of $9.46 \pm 11.7$ $\mathrm{pA}$, and $I_{\text {erase }} / I_{\text {program }}$ of $1.07 \times 10^{6}$ under 1000 consecutive sweep cycles. We also evaluated the dynamic behavior of the P-memory (Fig. S4). Stable switching behavior between the programmed and erased states was 


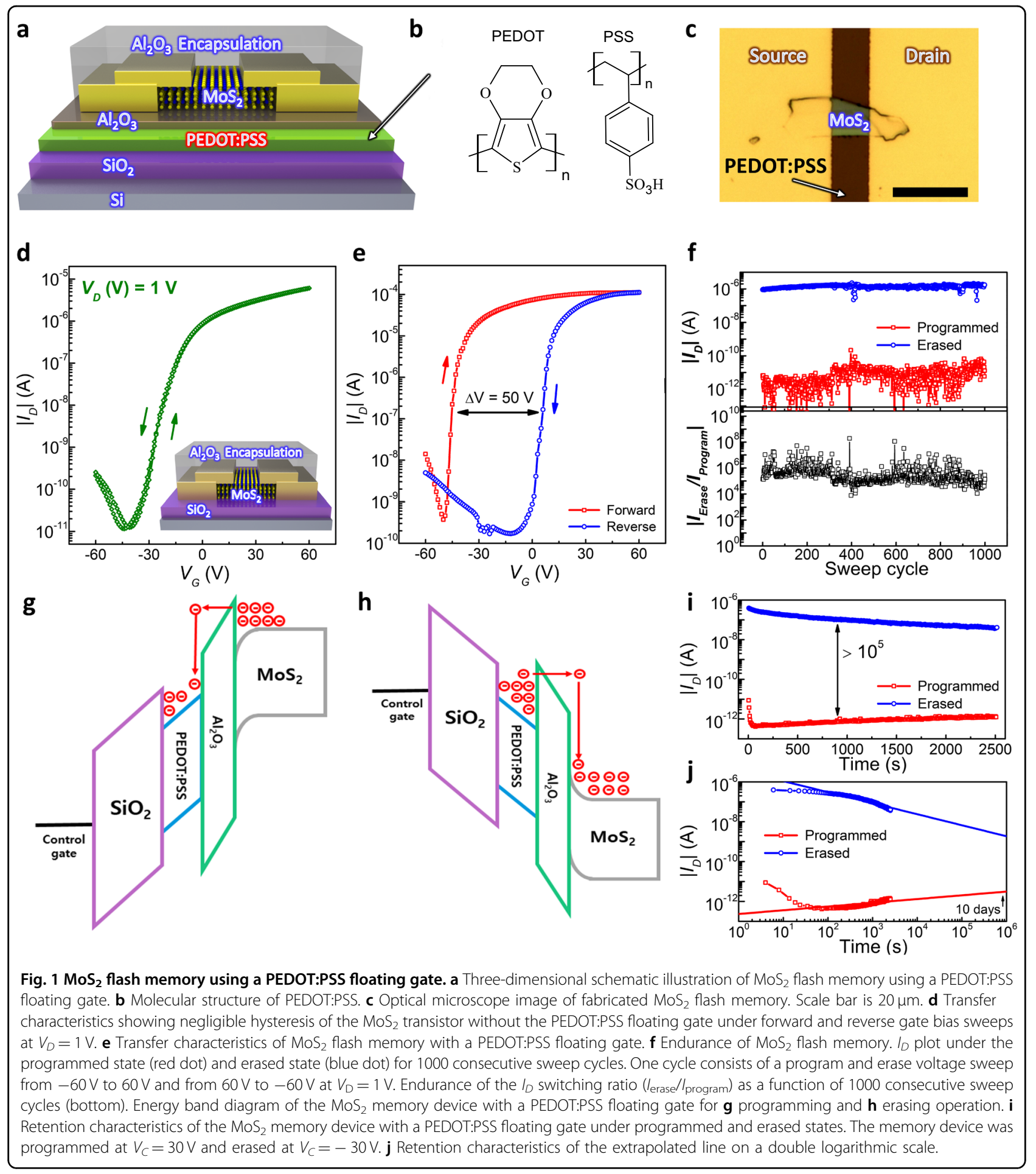

obtained depending on the applied voltage pulses, with a programming pulse of $45 \mathrm{~V}$ at $400 \mathrm{~ms}$ and an erasing pulse of $-45 \mathrm{~V}$ at $400 \mathrm{~ms}$ for a fixed $V_{D}=1 \mathrm{~V}$. Figure $1 \mathrm{~g}$, h shows simplified energy band diagrams of the P-memory under programming and erasing operations considering an energy bandgap of $\mathrm{MoS}_{2}$ of $5.15-5.39 \mathrm{eV}^{26,32}$. Electron carriers enter the PEDOT:PSS floating gate through the TDL $\mathrm{Al}_{2} \mathrm{O}_{3}$ layer by the mechanisms of Fowler-Nordheim tunneling ${ }^{33}$ and trap-assisted tunneling (TAT) ${ }^{34}$, resulting in a positive shift of $V_{T H}$. Under a negative applied $V_{G}$ (i.e., $V_{G}<-20 \mathrm{~V}$ ), the trapped electrons pass through the energy barrier inside the floating gate, causing a negative 


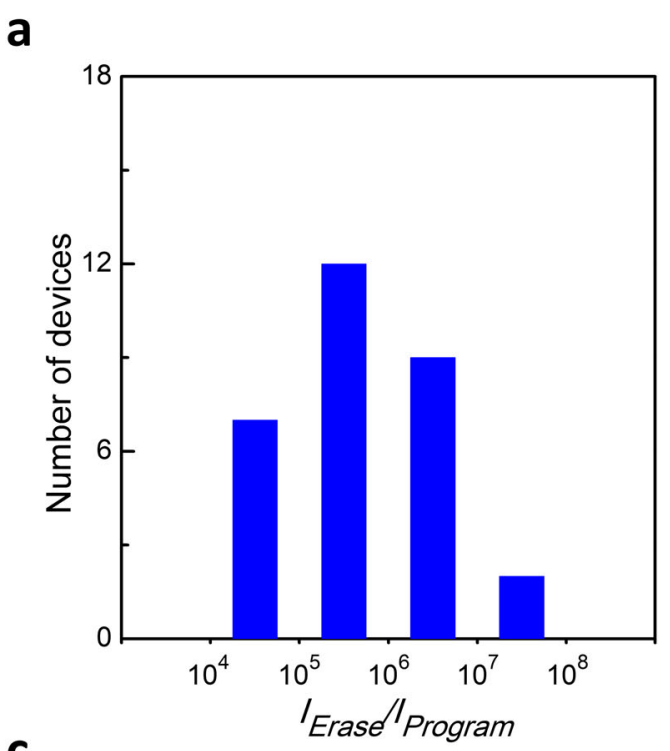

C

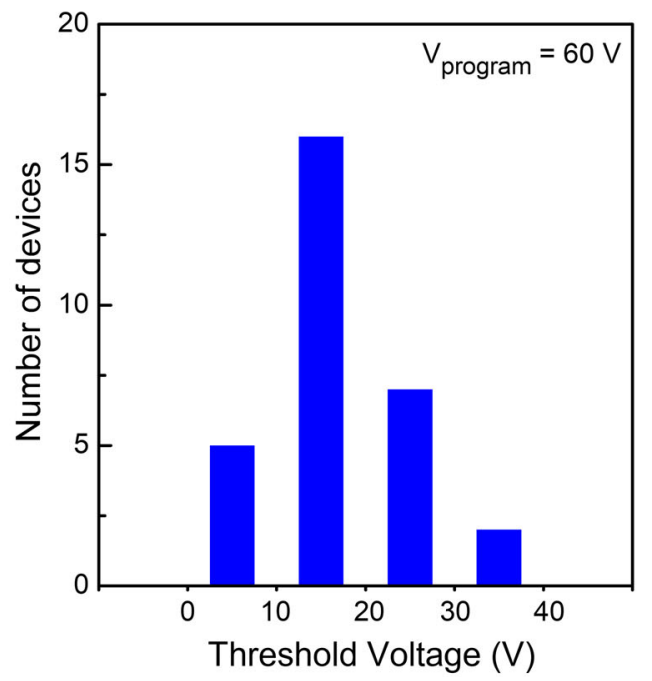

b

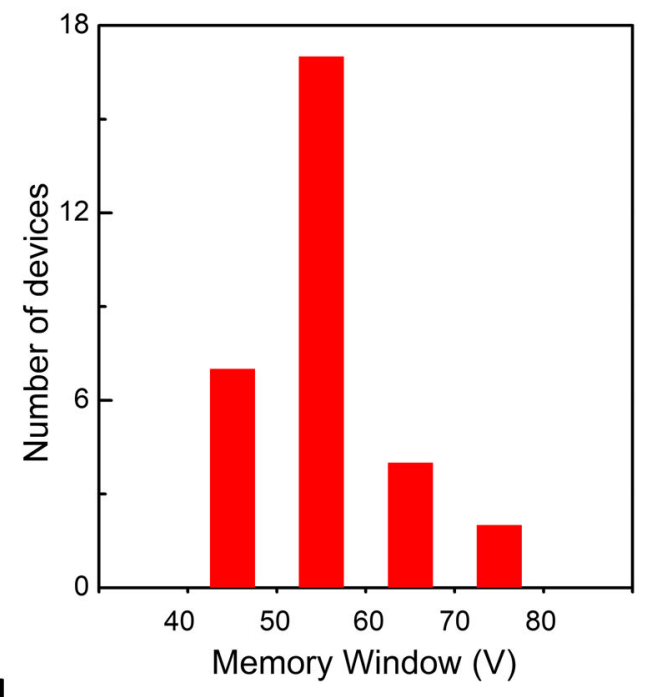

d

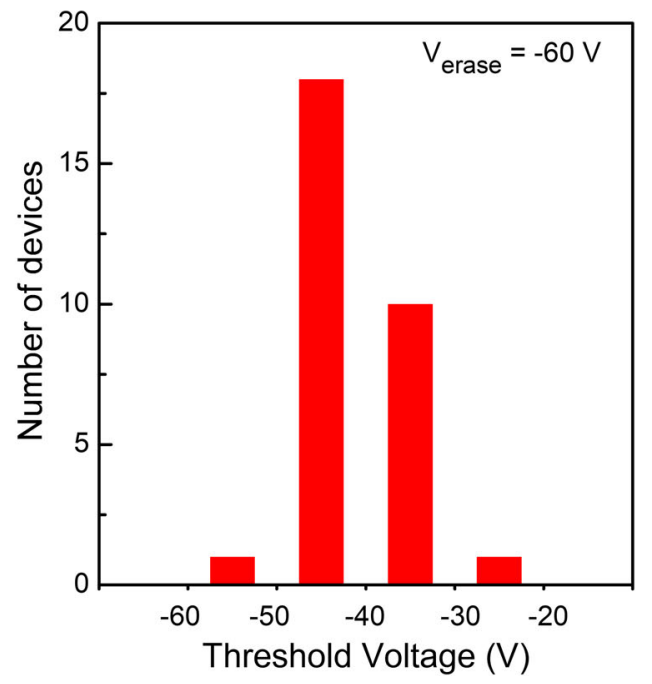

Fig. 2 Statistical analysis of the properties of $\mathbf{3 0}$ distinct MoS2 flash memories. Histogram showing the statistical distribution of a erase $/ /_{\text {program }}$ b the memory window, (c) the threshold voltage at $V_{\text {program }}=60 \mathrm{~V}$, and $\mathbf{d}$ the threshold voltage at $V_{\text {erase }}=-60 \mathrm{~V}$.

shift of $V_{T H}$. By enlarging the applied CG voltage $V_{C G}$ bias in the P-memory, the degree of $V_{T H}$ shift was controlled (Fig. S5). With greater applied $V_{G}$ and thus energy band bending to inject more electron carriers into the floating gate, a larger shift of $V_{T H}$ can be obtained.

To investigate the reproducibility and uniformity of the P-memory, we fabricated and characterized 30 devices. Figure 2a, b shows the statistical variation of the $I_{\text {program }} /$ $I_{\text {erase }}$ ratio (average value $=2.18 \times 10^{6} \pm 4.77 \times 10^{6}$ ) and the memory window (average value $=54.6 \mathrm{~V} \pm 7.80$ ), respectively, in the 30 devices. The distribution shows that the devices with an $I_{\text {program }} / I_{\text {erase }}$ ratio $\geq 10^{5}$ comprise $\sim 75 \%$ of the 30 devices. In addition, 17 devices have a memory window ranging from $50 \mathrm{~V}$ to $60 \mathrm{~V}, \sim 50 \%$ of the total. Figure 2c, d presents the $V_{T H}$ distribution in the programmed state $\left(V_{\text {program }}=60 \mathrm{~V}\right)$ and erased state $\left(V_{\text {erase }}=-60 \mathrm{~V}\right)$, respectively. The obtained average $V_{T H}$ is $16.40 \pm 8.25 \mathrm{~V}$ in the programmed state and $-40.53 \pm$ $5.87 \mathrm{~V}$ in the erased state. Details of the $30 \mathrm{P}$-memory device characteristics are given in Figs. S6 and S7.

\section{Photoactivation effect on the P-memory}

In the P-memory, the illumination light wavelength and intensity information can be stored as multilayered $\mathrm{MoS}_{2}-$ and PEDOT:PSS-polymer-generated photoactivated excess carriers (Fig. 3a, b). To study the photoactivation effect on the operation of the P-memory, we illuminated it with light (excitation wavelength $\lambda_{e x}=405 \mathrm{~nm}$ and incident 


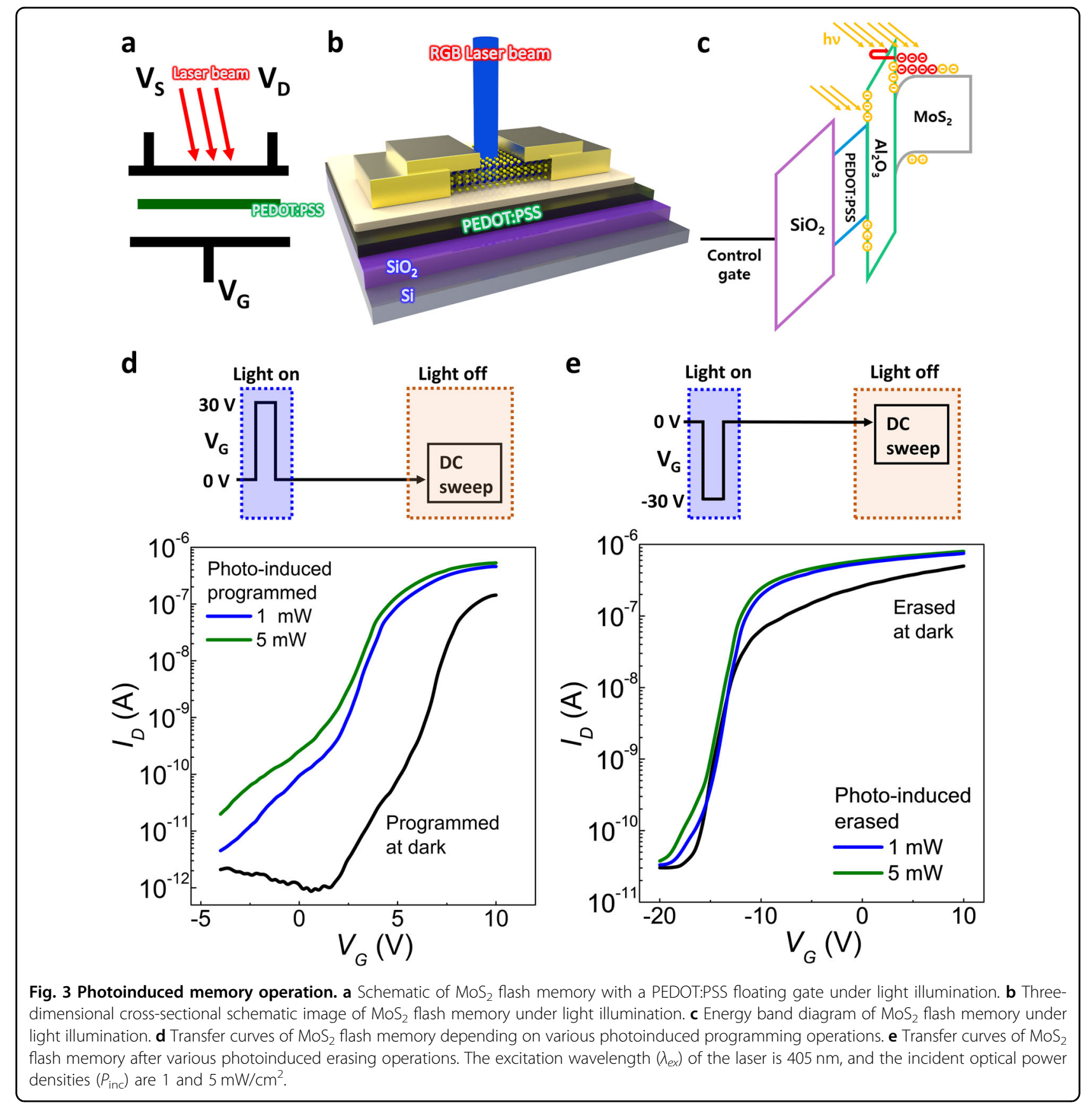

optical power densities $P_{i n c}=1$ and $5 \mathrm{~mW} / \mathrm{cm}^{2}$ ) during programming and erasing operations (i.e., photoinduced programming and photoinduced erasing, respectively). After the respective photoinduced programming and erasing operations, we performed a DC sweep of the Pmemory to observe how light illumination influences the charge programming and erasing behavior (Fig. 3d, e). When programming in the dark state was activated, $V_{T H}$ shifted to the positive side, showing $V_{T H}=7.18 \mathrm{~V}$, whereas when the programming was operated under a light illumination of $P_{i n c}=1 \mathrm{~mW} / \mathrm{cm}^{2}$, a smaller $V_{T H}$ shift to $V_{T H}=4.23 \mathrm{~V}$ was observed. The observed phenomenon is attributable to the charge screening effect of the photogenerated excess carriers, preventing tunneling of electron carriers into the floating PEDOT:PSS layer (Fig. 3c). As a result, a smaller $V_{T H}$ shift is induced by the programming under light illumination.

It was observed that a higher $P_{i n c}=5 \mathrm{~mW} / \mathrm{cm}^{2}$ resulted in more photogenerated excess carriers that contributed to the screening effect, and thus, less of a 

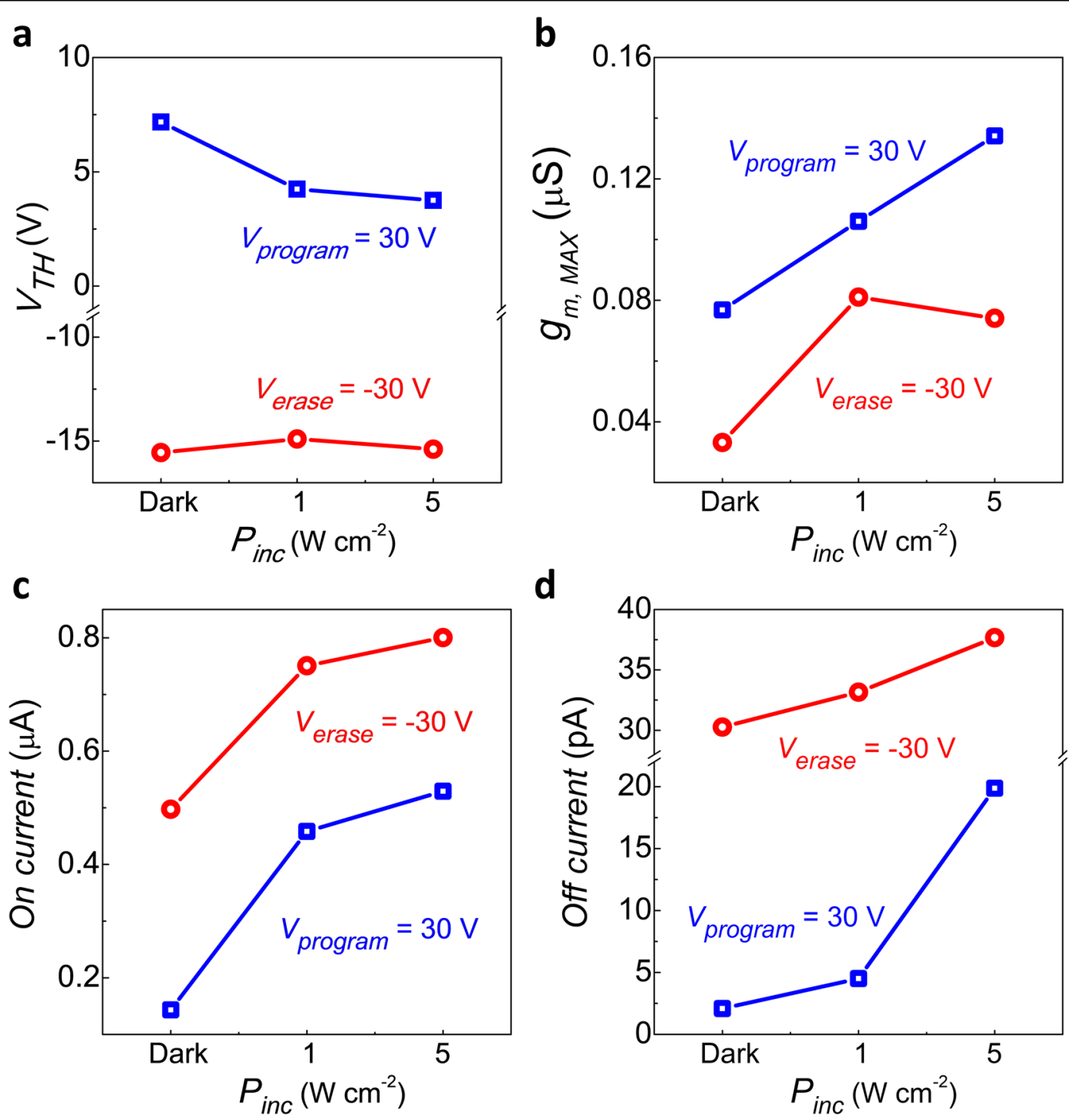

Fig. 4 Parameters extracted from photoinduced memory operation characteristics. Variation of the $\mathbf{a}$ threshold voltage $\left(V_{T H}\right), \mathbf{b}$ transconductance $\left(g_{\mathrm{m}}\right), \mathbf{c}$ on-current, and $\mathbf{d}$ off-current of $\mathrm{MoS}_{2}$ flash memory after various photoinduced programming/erasing operations. Red circles: erasing operation. Blue squares: programming operation.

$V_{T H}$ shift $\left(V_{T H}=3.75 \mathrm{~V}\right)$ was observed compared to that of the programmed state under $P_{i n c}=1 \mathrm{~mW} / \mathrm{cm}^{2}$ (Fig. 4a). We also investigated the light illumination effects during the erasing operation. Different from the photoinduced programming behavior, there was no $V_{T H}$ change under the photoinduced erasing operation. Rather than a change in the $V_{T H}$ shift, the photoinduced erasing operation resulted in an increase in the on-current in the switching-on region $\left(V_{G}>10 \mathrm{~V}\right)$. The observed increase in the on-current resulted from the slow decay of the generated photocurrent in the multilayered $\mathrm{MoS}_{2}$, which supports previously reported phenomena in several studies ${ }^{35-37}$. The photoinduced programming and erasing behaviors under other light illumination $\left(\lambda_{e x}=532\right.$ and $\left.638 \mathrm{~nm}\right)$ are shown in Figs. S8-11.

\section{Photosensing memory operation}

The photoinduced programming behavior with a change in $V_{T H}$ shift allowed us to demonstrate multilevel photomemory applications that combine photodiodes with memory functions. During the programming operation, the P-memory is programmed differently depending on the presence or absence of light illumination and its light intensity, and thus, the degree of light exposure can be directly stored in the device. We illuminated blue light $\left(\lambda_{e x}=405 \mathrm{~nm}\right)$ onto the P-memory during the programming operation at $V_{P}=30 \mathrm{~V}$, and we 


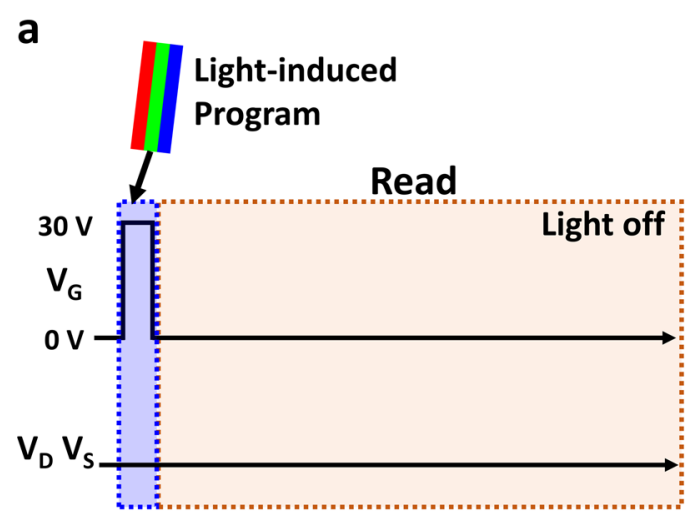

C

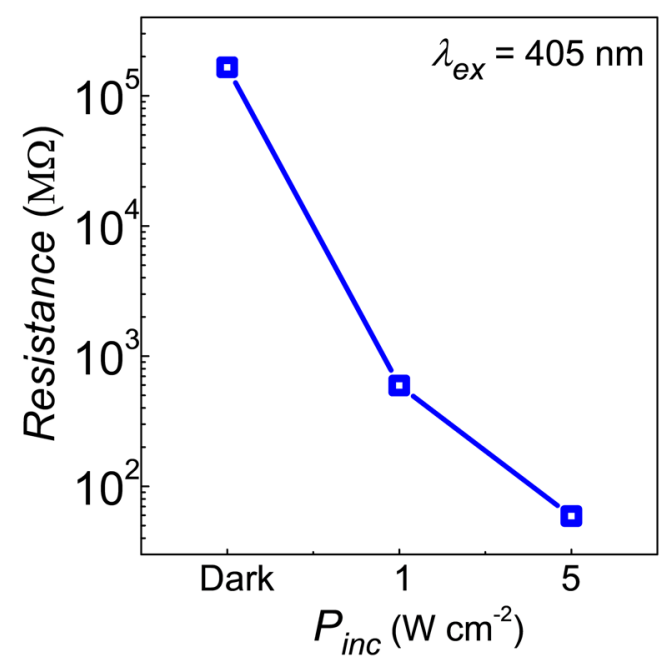

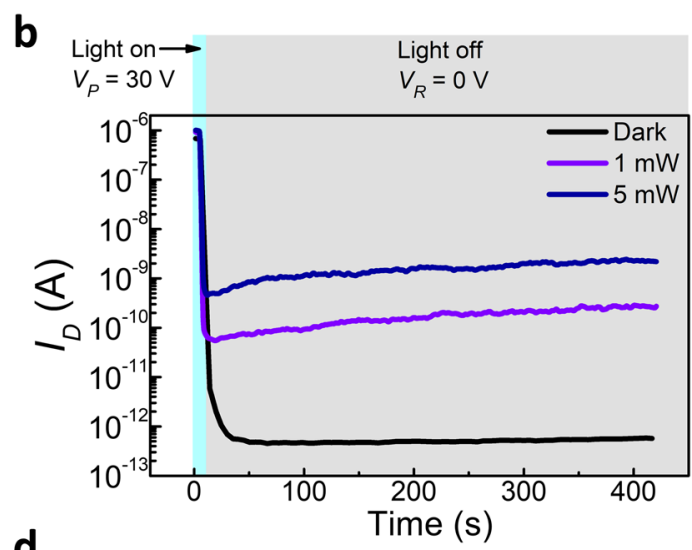

d

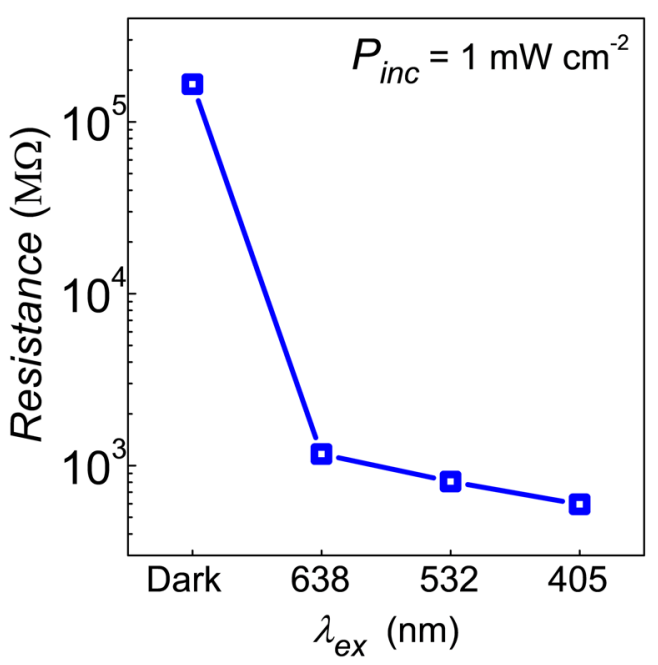

Fig. 5 Multilevel data storage characteristics of MoS2 flash memory under various photoinduced programming conditions. a Schematic diagram of measurement conditions depending on various light illumination under programming operation. $\mathbf{b}$ Multilevel retention characteristics of blue photoinduced programming with different light intensities at $V_{D}=0.1 \mathrm{~V}$. c Average resistance in the retention measurement until $400 \mathrm{~s}$ at $V_{D}=$ $0.1 \mathrm{~V}$ as a function of the incident power density $\left(P_{\text {inc }}\right)$. $\mathbf{d}$ Average resistance in red, green, and blue retention measurements until $400 \mathrm{~s}$ at $V_{D}=0.1 \mathrm{~V}$ as a function of the excitation wavelength $\left(\lambda_{e x}\right)$.

sequentially measured the read current at $V_{R}=0 \mathrm{~V}$ as a function of the photosensing retention time (Fig. 5a). The programming operation at $V_{P}=30 \mathrm{~V}$ in the dark resulted in the P-memory exhibiting $V_{T H}=7.18 \mathrm{~V}$, which produced a current of $1-2 \mathrm{pA}\left(I_{R}\right)$ at $V_{R}=0 \mathrm{~V}$ across the entire range of the photosensing retention time. On the other hand, the programming operation at $V_{P}=30 \mathrm{~V}$ under light illumination enabled the P-memory to provide a more than thousandfold higher $I_{R}$ than that in the programmed state in the dark. The increased $I_{R}$ with photoactivated programming was unchanged over the entire range of the photosensing retention time test ( $>400 \mathrm{~s}$ ) (Fig. 5b). A higher light intensity resulted in a higher $I_{R}$ with a lower resistance state of the P-memory (Fig. 5c). Under red and green light illumination $\left(\lambda_{e x}=\right.$ 532 and $638 \mathrm{~nm}$ ), we also observed similar $I_{R}$ increases. As expected, light with a lower wavelength enabled the Pmemory to have a greater $I_{R}$ increase with a lower resistance state, which potentially provides a classification operation according to the wavelength of light (Fig. 5d, Figs. S12 and S13).

\section{Mechanically flexible device manufacturing of the P- memory}

As the $\mathrm{MoS}_{2}$ and PEDOT:PSS layers can be manufactured on top of flexible substrates owing to their excellent thermal budget $\left(<200^{\circ} \mathrm{C}\right)$, we next implemented flexible P-memory. A solution-based PI film was coated on a $\mathrm{Si} / \mathrm{SiO}_{2}$ substrate, and flexible P-memory was fabricated with the same process as for the rigid memory (Scheme 1). For the BDL and the TDL, we deposited $100 \mathrm{~nm}$ - and $80 \mathrm{~nm}$-thick $\mathrm{Al}_{2} \mathrm{O}_{3}$, respectively. By delaminating the P-memory with the PI film from the substrate, we obtained flexible P-memory (Fig. 6a, b). Further details of the flexible P-memory fabrication are given in the Experimental Section. The flexible P-memory 

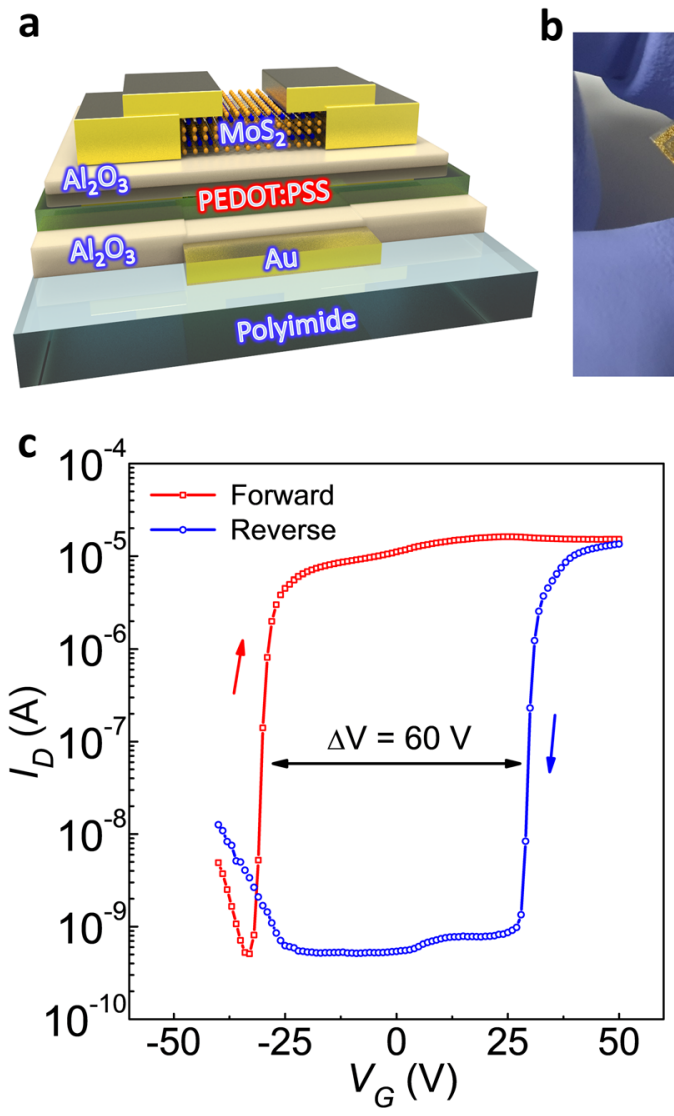

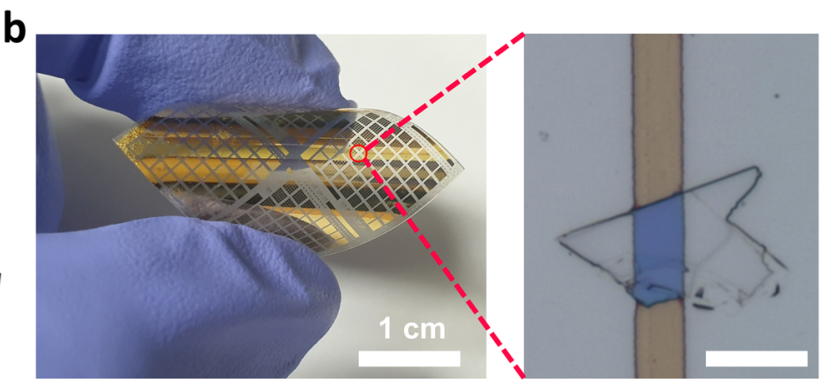

d

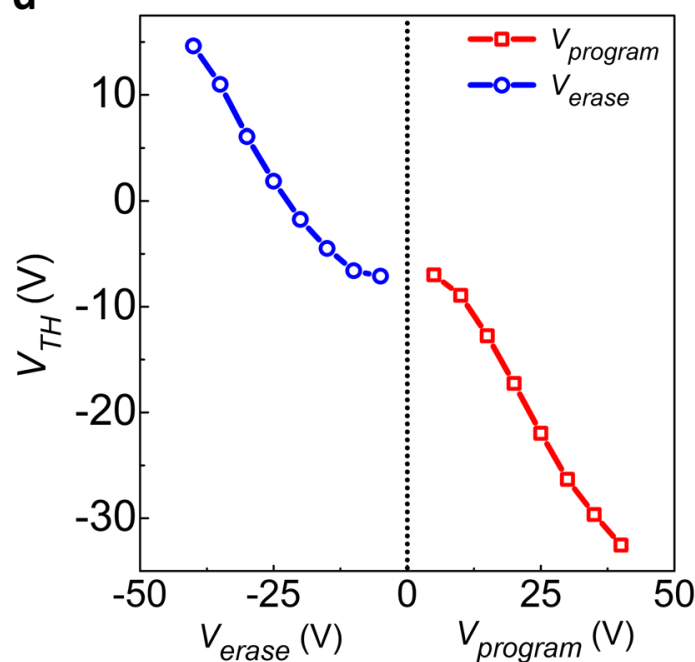

Fig. 6 Flexible MoS2 flash memory. a Three-dimensional schematic device structure of flexible MoS 2 flash memory on a PI substrate. b Photograph and $\mathrm{OM}$ image of fabricated flexible $\mathrm{MoS}_{2}$ flash memory. Scale bars are $1 \mathrm{~cm}$ and $10 \mu \mathrm{m}$, respectively. c Transfer characteristics of flexible $\mathrm{MoS}_{2}$ flash memory. The obtained memory window is $\sim 60 \mathrm{~V}$. $\mathbf{d}$ Extracted threshold voltage $V_{T H}$ under different $V_{\text {program }}$ and $V_{\text {erase }}$ at $V_{D}=1 \mathrm{~V}$.

exhibited a large memory window, $\Delta \mathrm{V}=60 \mathrm{~V}$, obtained by programming/erasing the charges in the PEDOT:PSS floating layer, as we described in the P-memory operation part (Fig. 6c). The amount of $V_{T H}$ shift can be controlled by the applied $V_{C G}$ bias, in which a higher $V_{C G}$ bias results in a larger $V_{T H}$ shift toward the negative or positive side depending on $V_{\text {erase }}$ and $V_{\text {program }}$, respectively.

To evaluate the mechanical durability of the flexible Pmemory, we measured its $I-V$ transfer characteristics before and after 1000 bending cycles at a bending radius of curvature $r=5 \mathrm{~mm}$ (Fig. 7a, Supporting Video 1). Details of the bending test are given in the Experimental Section. The flexible P-memory exhibited the same $I-V$ transfer characteristics without any degradation or current change compared to those before the 1000 bending cycles. The observed excellent mechanical durability resulted from the layered structure of $\mathrm{MoS}_{2}$ and the high flexibility of the PEDOT:PSS layer. To further investigate whether the flexible P-memory operated effectively after 1000 bending cycles, we performed a memory retention test (Fig. 7c). The current ratio of the programmed and erased states was above hundred for $8000 \mathrm{~s}$ of the retention measurement time.
Photosensing memory operation of the flexible P-memory

We also tested the photosensing retention time of the flexible P-memory after 1000 bending cycles (Fig. 7d). As expected, lower wavelength light illumination under the programming operation $\left(V_{\text {program }}=35 \mathrm{~V}\right)$ allowed the device to exhibit a higher $I_{R}$, so the flexible P-memory provided distinguishable resistance states depending on the light exposure properties. The abovementioned comprehensive memory tests confirmed that flexible Pmemory exhibited no abnormality in the memory or photoactivated memory operations even after 1000 bending cycles.

\section{Conclusions}

In summary, we proposed P-memory that combines a layered $\mathrm{MoS}_{2}$ thin-film transistor structure with a PEDOT:PSS floating gate. Owing to the virtues of the $\mathrm{MoS}_{2}$ and PEDOT:PSS combined structure, the Pmemory exhibited high performance, including a high switching current ratio $>10^{7}$, a large memory window $>50 \mathrm{~V}$, excellent endurance $>1000$ cycles, and a long retention time $>2000 \mathrm{~s}$. These were confirmed by 
a
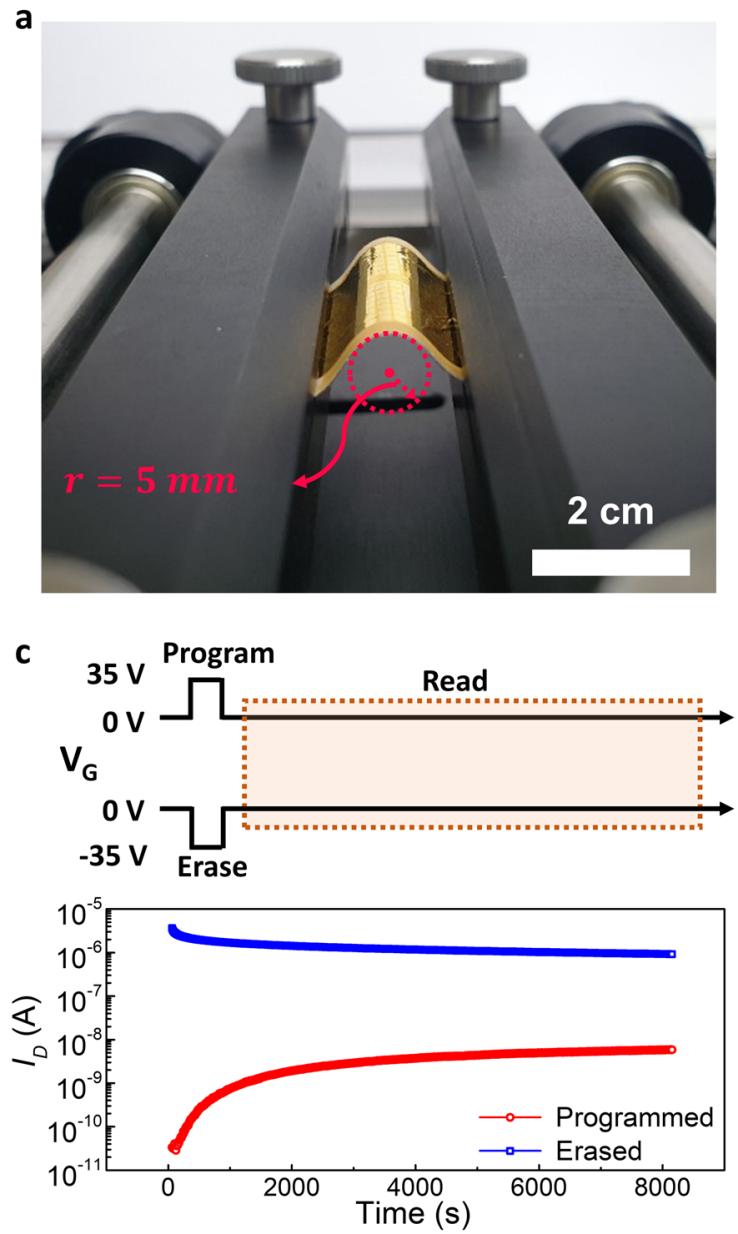

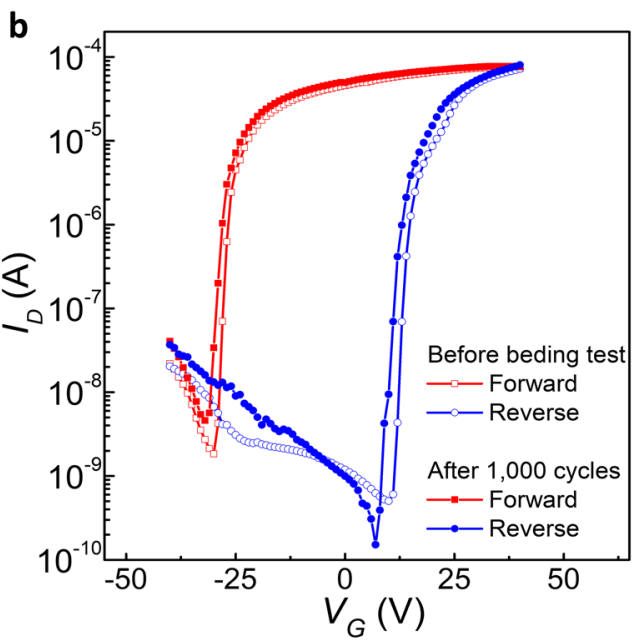

d

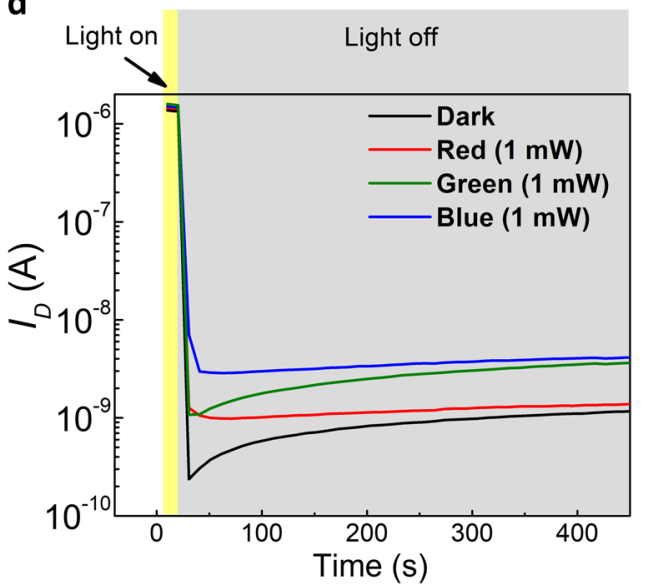

Fig. 7 Bending test and photoinduced operation of flexible MoS2 flash memory. a Photograph of MoS ${ }_{2}$ flash memory bent in the bending tester. The bending radius and scale bar are $5 \mathrm{~mm}$ and $2 \mathrm{~cm}$, respectively. $\mathbf{b}$ Comparison of transfer characteristics of MoS $\mathrm{S}_{2}$ flash memory before and after 1000 bending cycles with a $5 \mathrm{~mm}$ bending radius. c Schematic diagram of the retention measurement conditions and obtained retention characteristics of $\mathrm{MoS}_{2}$ flash memory under a control gate pulse of $\pm 35 \mathrm{~V}$ at $V_{\mathrm{D}}=0.1 \mathrm{~V}$ for $8000 \mathrm{~s}$ after 1000 bending cycles with a $5 \mathrm{~mm}$ bending radius. d Multilevel retention characteristics of red, green, and blue photoinduced programming $(638 \mathrm{~nm}, 532 \mathrm{~nm}$, and $405 \mathrm{~nm}$, respectively).

multiple device fabrication and characterization to evaluate the reproducibility and uniformity ( $>30$ devices). We also achieved a photoactivated memory operation that combines photodiode and memory functions. Depending on whether light was illuminated, the P-memory provided a different degree of programming, and thus, the degree of light exposure could be stored directly in the device. Furthermore, we demonstrated flexible P-memory by manufacturing the device on a flexible PI substrate. The results indicated that the device maintained its multifunctional memory behavior even after 1000 bending cycles. Through comprehensive experiments, this study showed not only the memory characteristics of conventional floating-gate-based flash memory but also new versatility such as mechanical flexibility and photoactivated memory behavior. These reported results are expected to be useful guidelines in the development of new electronic device features using new nanomaterials.

\section{Acknowledgements}

This research was supported by the National Research Foundation of Korea (2018R1A2B2003558) and the Basic Science Research Program through the National Research Foundation of Korea (NRF), which is funded by the Ministry of Education (2020R111A1A01070907 and 2020R111A1A01073884). H.Y. acknowledges funding support from the National Research Foundation of Korea (NRF) grant funded by the Korean government (MSIT) (No. NRF2020M3A9E4104385 and 2020R1A2C1101647).

\section{Author details}

${ }^{1}$ School of Advanced Materials Science and Engineering Sungkyunkwan University, Sunwon 440-746, Korea. ${ }^{2}$ Korea Institute of Industrial Technology, Cheonan 31056, Republic of Korea. ${ }^{3}$ Department of Electronic Engineering Gachon University, 1342 Seongnam-daero, Seongnam 13120, Korea

Conflict of interest

The authors declare no competing interests. 


\section{Publisher's note}

Springer Nature remains neutral with regard to jurisdictional claims in published maps and institutional affiliations.

Supplementary information The online version contains supplementary material available at https://doi.org/10.1038/s41427-021-00307-x.

Received: 10 January 2021 Revised: 19 February 2021 Accepted: 26 February 2021.

Published online: 30 April 2021

\section{References}

1. Huang, M. et al. Multifunctional high-performance van der Waals heterostructures. Nat. Nanotechnol. 12, 1148-1154 (2017).

2. Amani, M., Burke, R. A., Proie, R. M. \& Dubey, M. Flexible integrated circuits and multifunctional electronics based on single atomic layers of $\mathrm{MoS}_{2}$ and graphene. Nanotechnology 26, 115202 (2015).

3. Zhao, M. et al. A multifunctional and highly stretchable electronic device based on silver nanowire/wrap yarn composite for a wearable strain sensor and heater. J. Mater. Chem. C. 7, 13468-13476 (2019).

4. Zhang, L.-X. et al. Filter-Free Selective Light Monitoring by Organic Field-Effect Transistor Memories with a Tunable Blend Charge-Trapping Layer. ACS Appl. Mater. Interfaces 11, 40366-40371 (2019).

5. Han, J., Wang, W., Ying, J. \& Xie, W. Ambipolar organic thin-film transistorbased nano-floating-gate nonvolatile memory. Appl. Phys. Lett. 104, 2_1 (2014).

6. Kim, J. J., Cho, B., Kim, K. S., Lee, T. \& Jung, G. Y. Electrical characterization of unipolar organic resistive memory devices scaled down by a direct metaltransfer method. Adv. Mater. 23, 2104-2107 (2011).

7. Khan, M., Bhansali, U.S. \& Alshareef, H. N. High-performance non-volatile organic ferroelectric memory on banknotes. Adv. Mater. 24, 2165-2170 (2012).

8. Cho, B., Song, S., Ji, Y., Kim, T. W. \& Lee, T. Organic resistive memory devices: performance enhancement, integration, and advanced architectures. Adv. Funct. Mater. 21, 2806-2829 (2011).

9. Khan, Y. et al. Inkjet-printed flexible gold electrode arrays for bioelectronic interfaces. Adv. Funct. Mater. 26, 1004-1013 (2016).

10. $\mathrm{Xu}$, J. et al. Biodegradable natural pectin-based flexible multilevel resistive switching memory for transient electronics. Small 15, 1803970 (2019).

11. Zhang, P. et al. Structural Phase Transition Effect on Resistive Switching Behavior of $\mathrm{MoS}_{2}$-Polyvinylpyrrolidone Nanocomposites Films for Flexible Memory Devices. Small 12, 2077-2084 (2016).

12. Boni, G. A. et al. Multiple polarization states in symmetric ferroelectric heterostructures for multi-bit non-volatile memories. Nanoscale 9, 19271-19278 (2017).

13. Lan, Y.-W. et al. Nonvolatile molecular memory with the multilevel states based on $\mathrm{MoS}_{2}$ nanochannel field effect transistor through tuning gate voltage to control molecular configurations. Nanotechnology 31, 275204 (2020).

14. Cabaret, T. et al. Electro-grafted organic memristors: Properties and prospects for artificial neural networks based on STDP. 14th IEEE Int. Conf. Nanotechnol. 14, 499-504 (2014).
15. van de Burgt, Y. et al. A non-volatile organic electrochemical device as a lowvoltage artificial synapse for neuromorphic computing. Nat. Mater. 16 414-418 (2017).

16. Sangwan, V. K. et al. Multi-terminal memtransistors from polycrystalline monolayer molybdenum disulfide. Nature 554, 500-504 (2018).

17. $\mathrm{Lu}, \mathrm{X}$. et al. Large-area synthesis of monolayer and few-layer MoSe 2 films on $\mathrm{SiO}_{2}$ substrates. Nano Lett. 14, 2419-2425 (2014).

18. Yoo, $\mathrm{H}$. et al. Chemical doping effects on CVD-grown multilayer $\mathrm{MoSe}_{2}$ transistor. Adv. Electron. Mater. 4, 1700639 (2018).

19. Ahn, C. et al. Low-temperature synthesis of large-scale molybdenum disulfide thin films directly on a plastic substrate using plasma-enhanced chemical vapor deposition. Adv. Mater. 27, 5223-5229 (2015).

20. Kim, H. U. et al. Wafer-Scale and Low-Temperature Growth of 1T-WS2 Film for Efficient and Stable Hydrogen Evolution Reaction. Small 16, 1905000 (2020).

21. Zhou, J. et al. Synthesis of Co-Doped MoS 2 Monolayers with Enhanced Valley Splitting. Adv. Mater. 32, 1906536 (2020).

22. Hong, $\mathrm{X}$. et al. Ultrafast charge transfer in atomically thin $\mathrm{MoS}_{2} \mathrm{WS}_{2}$, heterostructures. Nat. Nanotechnol. 9, 682-686 (2014).

23. Zheng, W. et al. MoS 2 Van der Waals $p-n$ Junctions Enabling Highly Selective Room-Temperature $\mathrm{NO}_{2}$ Sensor. Adv. Funct. Mater. 30, 2000435 (2020).

24. Safeer, C. et al. Room-temperature spin Hall effect in graphene/MoS 2 van der Waals heterostructures. Nano Lett. 19, 1074-1082 (2019).

25. Feng, Z. et al. Chemical sensing by band modulation of a black phosphorus/ molybdenum diselenide van der Waals hetero-structure. 2D Mater. 3, 035021 (2016).

26. Choi, S., Shaolin, Z. \& Yang, W. Layer-number-dependent work function of $\mathrm{MoS}_{2}$ nanoflakes. J. Korean Phys. Soc. 64, 1550-1555 (2014).

27. Jadwiszczak, J. et al. $\mathrm{MoS}_{2}$ Memtransistors Fabricated by Localized Helium lon Beam Irradiation. ACS Nano 13, 14262-14273 (2019).

28. Wang, C. Y. et al. 2D Layered Materials for Memristive and Neuromorphic Applications. Adv. Electron. Mater. 6, 1901107 (2020).

29. Vélez, S. et al. Gate-tunable diode and photovoltaic effect in an organic-2D layered material p-n junction. Nanoscale 7, 15442-15449 (2015).

30. Huang, Y. et al. Van der Waals coupled organic molecules with monolayer $\mathrm{MoS}_{2}$ for fast response photodetectors with gate-tunable responsivity. ACS Nano 12, 4062-4073 (2018).

31. Lee, J. J., Lee, S. H., Kim, F. S., Choi, H. H. \& Kim, J. H. Simultaneous enhancement of the efficiency and stability of organic solar cells using PEDOT: PSS grafted with a PEGME buffer layer. Org. Electron. 26, 191-199 (2015).

32. Zhang, B. et al. Work function and band alignment of few-layer violet phosphorene. J. Mater. Chem. A 8, 8586-8592 (2020).

33. Cho, Y. et al. Design and Characterization of Semi-Floating-Gate Synaptic Transistor. Micromachines 10, 32 (2019).

34. Jiménez-Molinos, F., Palma, A., Gámiz, F., Banqueri, J. \& Lopez-Villanueva, J. Physical model for trap-assisted inelastic tunneling in metal-oxidesemiconductor structures. J. Appl Phys. 90, 3396-3404 (2001).

35. Kufer, D. \& Konstantatos, G. Highly sensitive, encapsulated $\mathrm{MoS}_{2}$ photodetector with gate controllable gain and speed. Nano Lett. 15, 7307-7313 (2015).

36. Zhang, Y. et al. Photothermoelectric and photovoltaic effects both present in $\mathrm{MoS}_{2}$. Sci. Rep. 5, 7938 (2015).

37. Zhang, W. et al. High-gain phototransistors based on a CVD MoS 2 monolayer. Adv. Mater. 25, 3456-3461 (2013). 